\title{
Isolated Duodenal Trauma following Cricket Ball Injury
}

\author{
${ }_{1}^{1}$ Gautham Krishnamurthy, ${ }^{2}$ Hemanth Kumar, ${ }^{3}$ Rinki Das, ${ }^{4}$ Rajesh Gupta
}

\begin{abstract}
A fifteen years old boy presented to the emergency department with blunt trauma abdomen following cricket ball injury. At the time of presentation, patient was hemodynamically stable with diffuse abdominal tenderness and right hypochondrial guarding. On exploratory laparotomy, he was found to have isolated duodenal laceration and underwent pancreas preserving total duodenectomy with ampullojejunostomy. In the postoperative period, patient developed fever with respiratory tract infection and eventually succumbed to multiorgan dysfunction. To the best of our knowledge, this is the first case report describing isolated duodenal injury resulting from blunt abdominal trauma due to cricket ball.
\end{abstract}

Keywords: Ampullojejunostomy, Cricket ball injury, Pancreas preserving total duodenectomy.

How to cite this article: Krishnamurthy $G$, Kumar H, Das R, Gupta R. Isolated Duodenal Trauma following Cricket Ball Injury. J Postgrad Med Edu Res 2015;49(4):213-215.

\section{Source of support: Nil}

Conflict of interest: None

\section{INTRODUCTION}

Cricket is considered a relatively safer sport when compared to adventurous motor sports. Despite increase in the safety and efficacy of the protection devices, morbidity and even mortality is possible. Most of the injuries suffered by cricketers are limb injuries. ${ }^{1}$ Blunt abdominal trauma due to cricket ball leading to injuries to intraabdominal organs has been reported., ${ }^{2,3}$ But isolated duodenal injury due to cricket ball has not been reported before. We present a case of high grade duodenal injury caused by cricket ball requiring surgical intervention.

\section{CASE REPORT}

A 15 years old male presented to the emergency department 4 hours after sustaining abdominal injury by a cricket ball. At the time of presentation, patient gave history of abdominal pain over the right upper quadrant

\footnotetext{
${ }^{1-3}$ Senior Resident, ${ }^{4}$ Professor

${ }^{1-4}$ Division of Surgical Gastroenterology, Department of General Surgery, Postgraduate Institute of Medical Education and Research, Chandigarh, India

Corresponding Author: Rajesh Gupta, Professor, Division of Surgical Gastroenterology, Department of General Surgery Postgraduate Institute of Medical Education and Research Chandigarh, India, Phone: 9216512636, e-mail: rajsarakshi@ yahoo.co.in
}

and two episodes of nonbilious vomiting. On examination, he was having tachycardia and hypotension which responded to fluid resuscitation. Abdomen was distended with diffuse tenderness and right hypochondrial guarding. Chest Roentgenogram showed free air under diaphragm. Computed tomography (CT) showed duodenal injury with contrast extravasation into the general peritoneal cavity (Fig. 1). After optimization, patient underwent exploratory laparotomy. There was 2 liters of biliopurulent fluid in the peritoneal cavity with a $4 \times 5 \mathrm{~cm}$ laceration in the second part of the duodenum involving more than $50 \%$ of the circumference. Ampulla was intact. Considering the young age and early presentation, pancreas preserving duodenectomy was done. Reconstruction included ampullojejunostomy with transanastomotic biliary and pancreatic stent and gastrojejunostomy (Figs 2A to C). Feeding jejunostomy was performed for postoperative nutritional management. Postoperatively patient was hemodynamically stable requiring mechanical ventilation. Patient developed high grade fever on second postoperative day. Chest X-ray showed bilateral minimal infiltrate. Feeding jejunostomy feeds were started on 3rd postoperative period. Antibiotics was changed as per the intraoperative culture and endotracheal aspirate culture. The respiratory signs were decreasing despite the fever persisting. Computed tomography of the abdomen for evaluation of fever showed no evidence of collection and intact anastomosis. Pancreas was normal. On 10th postoperative day, wound dehiscence

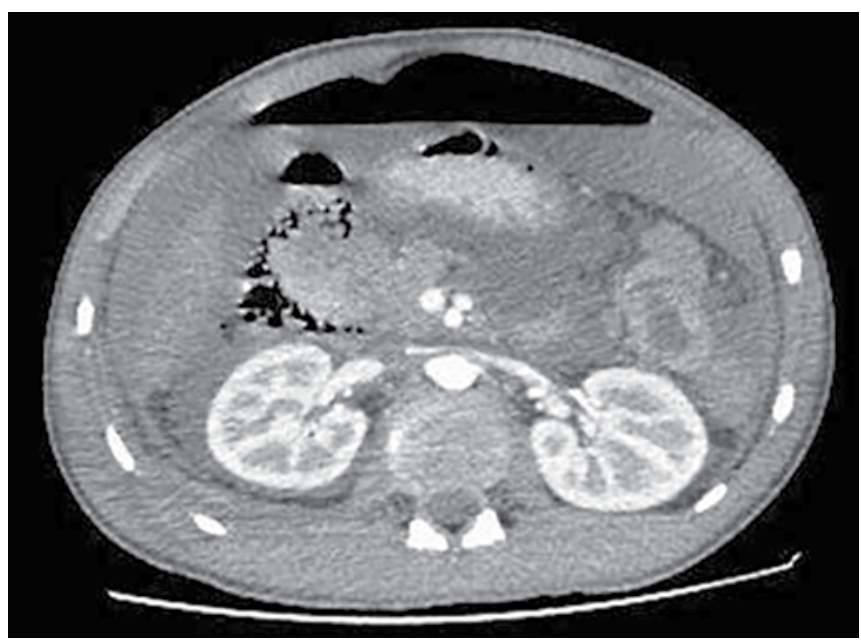

Fig. 1: Computed tomography of abdomen showing contrast extravasation from duodenum, air foci around duodenum and gross pneumoperitoneum 


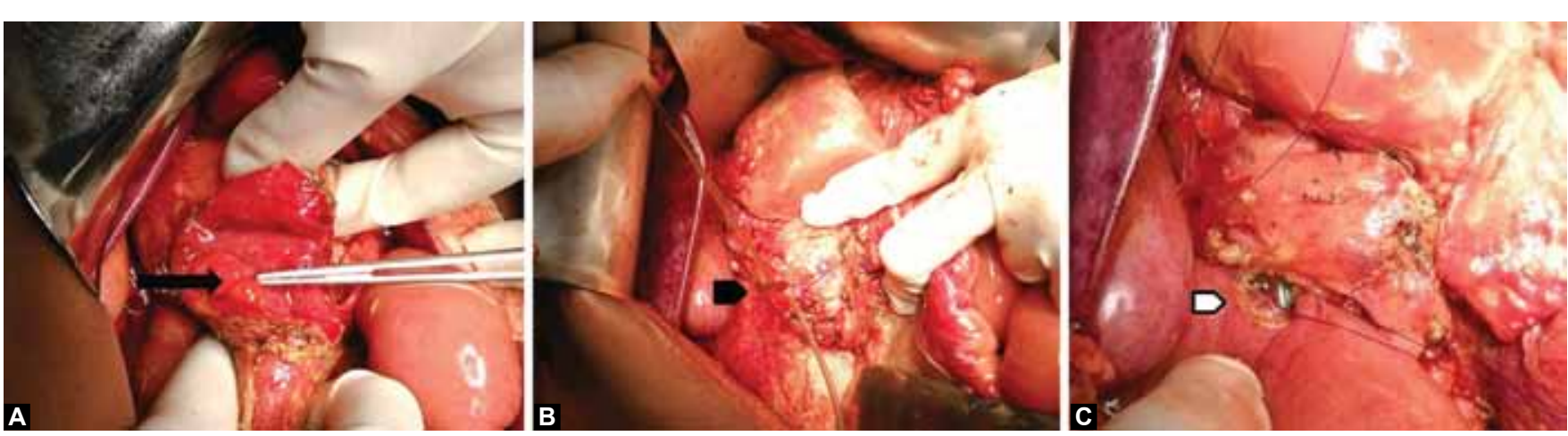

Figs 2A to C: (A) Duodenal laceration at the level D2 exposing the ampulla (Black arrow), (B) transbiliary and transpancreatic stents being placed at the ampulla (black arrowhead), (C) ampullojejunostomy (white arrowhead)

with purulent discharge was noted and irrigation initiated through the defect. However, patient developed multiorgan dysfunction and eventually succumbed to it on 11th postoperative day.

\section{DISCUSSION}

Duodenal injuries occur in $2 \%$ of all blunt trauma abdomen. ${ }^{4}$ The mechanism of injury involves an anteroposterior compressive force resulting from a steering wheel, handle bar or seat belt crushing the duodenum against the spine or posterior abdominal wall. ${ }^{5}$ Rarely, it can also be due to tangential shearing force against a relatively immobile duodenum or rupture due to increased intraluminal pressure. ${ }^{5,6}$ To the best of our knowledge, this is the first case report describing isolated duodenal injury resulting from blunt abdominal trauma due to cricket ball.

Duodenal injuries include a wide spectrum starting from contusion or intramural hematoma (Grade 1) to complete devascularization (Grade 5). Duodenal laceration (Grades 2 to 4 ) lies in the middle of this spectrum with ampullary involvement forming a separate entity (Grade 4). ${ }^{7}$ Duodenal injuries are associated with high morbidity (12-63\%) and mortality ranges from 6 to $29 \%{ }^{8}$

After full thickness duodenal injury, the contamination is initially confined to the retroperitoneum, not associated with obvious abdominal signs. Then with further contamination, the lesser sac, and then the greater sac are involved causing generalized peritonitis. ${ }^{9}$ The management of duodenal injuries is a challenging surgical task given the high incidence of associated other intraabdominal injuries, diagnostic delay and the complex anatomy.

Imaging plays a crucial role in diagnosis as even high grade injuries can present with minimal abdominal signs. Findings suggestive of duodenal injury in CT include extraluminal gas in the retroperitoneum, discontinuity of the duodenal wall, and positive contrast extravasation. ${ }^{9}$

The varied degree of injury and the numerous surgical options makes it difficult to standardize the management of duodenal injury. Common surgical procedures for management of duodenal injuries include primary closure after debridement, resection and anastomosis, duodenal diverticularization, pyloric exclusion and even pancreaticoduodenectomy. ${ }^{10}$ None of the surgical repair is immune from developing duodenal fistula. ${ }^{11}$ The debate regarding the need for pyloric exclusion for all cases of duodenal injuries has been settled with studies indicating no difference in outcome between primary repair and pyloric exclusion even in high grade duodenal injuries. ${ }^{12}$ Blocksom et al found that the infectious complications in duodenal injuries were increased when there was an associated abdominal arterial injury, injury severity score of greater than 25 , associated pancreatic injury, and lowest or core body temperature $<35^{\circ} \mathrm{C} .{ }^{13} \mathrm{In}$ the era of damage control surgery, staged surgeries have also gained popularity with promising results. ${ }^{14}$

Pancreas preserving total duodenectomy in complex isolated duodenal injuries can be suitable alternative to pancreaticoduodenectomy with the advantage of avoiding pancreatic resection and anastomosis in undilated bile or pancreatic duct. In circumstances of associated pancreatic head disruption staged Whipples procedure can be a suitable option. Though the technique has been widely done in elective setting for specific tumors of duodenum, case series in trauma has also been described with successful outcome. ${ }^{15}$ The reconstruction following resection can be done either with invagination pancreaticojejunostomy, ampullojejunostomy after papillotomy or without enteric anastomosis. ${ }^{16,17}$ In our cases, despite early surgery and intact anastomosis, the patient succumbed to sepsis resulting from initial peritonitis.

\section{CONCLUSION}

Information about the mechanism of injury and physical examination is valuable to suspect duodenal injury. Surgical management has to be tailored according to the hemodynamic status, associated other organ injury, grade of duodenal injury and technical expertise. Pancreas preserving total duodenectomy is a feasible option for isolated high grade duodenal injury. 


\section{REFERENCES}

1. Stretch RA, Orchard J. Cricket injuries: a longitudinal study of the nature of injuries to South African cricketers. Br J Sports Med 2003 Jun;37(3):250-253.

2. Philipoff AC, Rowcroft A, Weber DG. Novel presentation of a cricket ball-related intra-abdominal injury: genitofemoral nerve referred pain. BMJ Case Rep 2015 Aug;2015: doi: 10.1136/bcr2014-208024.

3. du Toit DF, Rademan F. Splenic rupture caused by a cricket ball. A case report. S Afr Med J 1987 Jun;71(12):796.

4. Linsenmaier U, Wirth S, Reiser M, Korner M. Diagnosis and classification of pancreatic and duodenal injuries in emergency radiology. Radiograph 2008 Oct;28(6):1591-1602.

5. Smiley K, Wright T, Skinner S, Iocono JA, Draus JM. Primary closure without diversion in management of operative blunt duodenal trauma in children. ISRN Pediatr 2012;2012: 298753.

6. Nasr EN, Bhatti MA, Warner E. Management of isolated blunt duodenal injury. J Natl Med Assoc 1981 Jun;73(6):525-529.

7. Moore EE, Cogbill TH, Malangoni MA, Jurkovich GJ, Champion HR, Gennarelli TA, et al. Organ injury scaling, II: Pancreas, duodenum, small bowel, colon, and rectum. J Trauma 1990 Nov;30(11):1427-1429.

8. Bozkurt B, Ozdemir BA, Kocer B, Unal B, Dolapci M, Cengiz O. Operative approach in traumatic injuries of the duodenum. Acta Chir Belg 2006 Jul-Aug;106(4):405-408.
9. Melamud K, LeBedis CA, Soto JA. Imaging of pancreatic and duodenal trauma. Radiol Clin North Am 2015 Jul;53(4): 757-771.

10. Carrillo EH, Richardson JD, Miller FB. Evolution in the management of duodenal injuries. J Trauma 1996 Jun;40(6):1037-1045.

11. Degiannis E, Boffard K. Duodenal injuries. Br J Surg 2000 Nov; 87(11):1473-1479.

12. Seamon MJ, Pieri PG, Fisher CA, Gaughan J, Santora TA, Pathak AS, et al. A 10-year retrospective review: does pyloric exclusion improve clinical outcome after penetrating duodenal and combined pancreaticoduodenal injuries? J Trauma 2007 Apr;62(4):829-833.

13. Blocksom JM, Tyburski JG, Sohn RL, Williams M, Harvey E, Steffes $\mathrm{CP}$, et al. Prognostic determinants in duodenal injuries. Am Surg 2004 Mar;70(3):248-255.

14. Rickard MJ, Brohi K, Bautz PC. Pancreatic and duodenal injuries: keep it simple. ANZ J Surg 2005 Jul;75(7):581-586.

15. Wig JD, Kudari A, Yadav TD, Doley RP, Bharathy KG, Kalra N. Pancreas preserving total duodenectomy for complex duodenal injury. JOP 2009 Jul 6;10(4):425-428.

16. Imamura M, Komoto I, Doi R, Onodera H, Kobayashi H, Kawai Y. New pancreas-preserving total duodenectomy technique. World J Surg 2005 Feb;29(2):203-207.

17. Koshariya M, Jagad RB, Kawamoto J, Papastratis P, Kefalourous $\mathrm{H}$, Porfiris $\mathrm{T}$, et al. Pancreas-preserving total duodenectomy without pancreato-enteric anastomosis. Hepatogastroenterol 2007 Oct-Nov;54(79):2123-2128. 